\title{
MiRNA in atopic dermatitis
}

\author{
Michał Rożalski, Lidia Rudnicka, Zbigniew Samochocki \\ Department of Dermatology, Medical University of Warsaw, Warsaw, Poland
}

Adv Dermatol Allergol 2016; XXXIII (3): 157-162

DOI: 10.5114/ada.2016.60606

\begin{abstract}
MicroRNAs are relatively new molecules that have been widely studied in recent years as to determine their exact function in the human body. It is suggested that microRNAs control approx. $30 \%$ of all genes, making them one of the largest groups that control the expression of proteins. Various functions of miRNAs have already been described. In skin diseases, there are more and more studies describing an altered expression of microRNAs in the skin or serum. Relatively little is known about the function of these molecules in atopic dermatitis, which prompted us to gather current reports on this subject.
\end{abstract}

Key words: atopic dermatitis, miRNA, microRNA, single-stranded RNA.

Innate and acquired immunity of the organism is under constant control of genes undergoing dynamic changes in expression. One of the factors that influence this phenomenon are non-coding RNAs - microRNAs (miRNAs). MiRNAs were discovered in 1993 by Victor Ambros in the nematode Caenorhabditis elegans as a molecule inhibiting the expression of genes that affect the transformation from the larval to mature form [1]. Lin-4 was the first described miRNA, followed by the description of let-7 [1]. The following years led to the detection of miRNAs in fruit flies, plants and animals, and now miRNAs are widely studied in all branches of medicine [2].

MiRNAs are short single-stranded RNA molecules built of 19-25 nucleotides. Like the mRNAs (messenger RNA), miRNAs are formed in the nucleus through transcription by means of RNA polymerase II. Initially, they create a longer transcript called pri-miRNAs. These molecules can be produced by independent promoters as polycistronic transcripts or they might be embedded in introns of protein-coding genes. Pri-miRNAs are cleaved by RNase III - endonuclease type Drosha-DGCR8 (DiGeorge syndrome critical region gene 8) complex to small hairpin-like precursors which are called pre-miRNAs. The particles are transported from the nucleus to the cytoplasm by exportin-5-RAN-GTP complex. In the cytoplasm pre-miRNAs are further cleaved by enzyme Dicer, which results in a short RNA duplex. One strand of such a mol- ecule is incorporated into RNA-induced silencing complex (RISC) which contains one of four proteins Argonaute (AGO) and trinucleotide repeat-containing protein 6 (TNRC6), otherwise known as glycine-tryptophan 182kDA protein (TNRC6) $[3,4]$. In this complex miRNAs are bound to the region of $3^{\prime}$ untranslated mRNAs. This connection covers at least 6-8 nucleotides in length. Thanks to these properties miRNA molecules cause gene silencing through either degradation or inhibition of mRNAs translation [5-7]. The expression of miRNAs is tissue and cell dependent, e.g. miR-146 expression is the highest in cells of the immune system, whereas expression of miR203 becomes greater in keratinocytes [8].

The number of miRNAs in the human genome is estimated at about $2500[9,10]$ and they are divided into 239 families [11-13]. One type of miRNA can influence expression of many genes by binding to different mRNAs. It is suggested that miRNAs control approx. $30 \%$ of all genes, making them one of the largest groups that control the expression of proteins [12]. Various functions of miRNAs have already been described, such as the impact on the proliferation and differentiation of cells, apoptosis, cellular stress response or influence on the immune system [14-16]. MiRNAs have recently been detected not only in cells but also in body fluids: serum, plasma, urine and saliva [17-21]. It has been shown that the concentration of

Address for correspondence: Michał Rożalski, Department of Dermatology, Medical University of Warsaw, 82A Koszykowa St, 02-008 Warsaw, Poland, phone: +48 791427 865, e-mail: michal_rozalski@vp.pl Received: 8.07.2015, accepted: 27.07.2015. 
miRNAs in serum reflects enhanced expression of these molecules in the body [22].

Recently published studies have shown altered expression of miRNAs in some inflammatory skin diseases, mainly in psoriasis and systemic sclerosis (SSc).

Psoriasis is the most common immune-mediated chronic inflammatory skin disease characterized by hyperproliferative keratinocytes and infiltration of T cells, dendritic cells, macrophages and neutrophils. Deregulation of immune cells in the skin plays a critical role in psoriasis development [23]. Increased expression of miR-203 and miR-146a has been demonstrated within active lesions as miR-203 suppresses SOCS-3 (cytokine signaling 3), which results in increased keratinocyte proliferation [8]. Impaired expression of miR-146a can affect the Th cells and monocyte-derived dendritic cells and increased concentration of TNF- $\alpha$, which can induce the severity of lesions [24]. Increased expression of miR-22, miR-24-1, miR-498 and miR-551a has also been demonstrated in the affected and unaffected skin of psoriasis patients compared to the healthy group [25]. In contrast, expression of miR-424 in patients with psoriasis has been significantly lower in the affected skin compared to the healthy control. Decreased expression of this molecule leads to increased expression of mitogen-activated protein kinase kinase 1 (MEK1) and cyclin E1 in keratinocytes, which stimulates the proliferation of these cells [26]. In the serum of psoriasis patients increased expression of miR-369-3p has been detected, which positively correlates with Psoriasis Area and Severity Index (PASI) [27].

Systemic sclerosis is a rare autoimmune disease characterized by pathological inflammation, fibrosis and vascular changes resulting in skin fibrosis and internal organs' manifestations [28]. MiRNAs are involved in the regulation of collagen synthesis and fibrosis. Systemic sclerosis patients show an increase in expression of miR-21 in the skin and fibroblasts [29], miR-92a in fibroblasts and serum [30] and miR-7 in fibroblasts [31]. These molecules influence Sma and Mad Related Family 7 (SMAD7) [29], matrix metalloproteinase-1 (MMP-1) [30] and expression of collagen [31], respectively, which may cause the severity of fibrosis. Reduction in expression of miR-145 in the skin and fibroblasts of patients with scleroderma by upregulation of SMAD and decrease in expression of miR-29b by increased expression of collagen type $1 \alpha 1$ (COL1A1) result in an increase in fibrosis [29]. What is more, reductions in expression of miR-29a and miR-196a result in an increase in collagen I synthesis by fibroblasts and enhanced fibrosis [32, 33].

\section{Atopic dermatitis}

The etiopathogenesis of atopic dermatitis (AD) is complex and not fully understood. It is believed that the disease is associated with the epidermal barrier damage and quantitative/functional innate and acquired immune system disorders. Patients with AD have shown a disorder in the balance of T lymphocytes, dysfunction in synthesis of cytokines, chemokines, total IgE, antimicrobial peptides, proteases and their inhibitors as well as in synthesis of proteins responsible for the correct structure of epithelial cells. It is developed in genetically predisposed people under the influence of various environmental factors [34, 35].

An estimated profile of miRNAs in the skin lesions of $A D$ patients has been determined by means of a microarray. Elevated expression of let-7i, miR-24, miR-27a, miR-29a, miR-193a, miR-199a, miR-222 has been found and, like in patients with psoriasis, miR-21, miR-146a, miR-20a, miR17-5p, miR-106b have also been elevated. Atopic dermatitis patients, as psoriatic patients, have been characterized by reduced expression of miR-122a, miR-133a-133b, miR-326, miR-215 and miR-133b, whereas reduced expression of miR-33, miR-483, miR-515-5p and miR-519d has occurred only in $\operatorname{AD}$ patients $[8,36]$.

Another study has identified 10 upregulated miRNAs in the changed AD patients' skin (miR-501, miR-223, miR-155, miR-135b, miR-142-3p, miR-142-5p, miR-362, miR-487b, miR-31, miR-187) and 34 downregulated ones (miR-204, miR-486, miR-375, miR-383, miR-10a, miR-125b, miR-149, miR-99a, miR-100, miR-328, miR-193b, miR-195, miR-23a, miR-452, let-7b, miR-197, miR-214, miR-30e-3p, let-7a, let-7c, miR-196b, miR-30a-3p, miR-26a, miR-26b, miR-101, miR-199b, let-7f, let-7d, miR-335, miR-143, miR145, miR-365, miR-196a, miR-615) [37].

MiR-155 molecule is involved in regulation of the innate and acquired immune response, immune cell development and carcinogenesis [38]. T cells, dendritic cells, fibroblasts, and mast cells in the skin show the greatest expression of this molecule [37].

Patients with $A D$ have very high expression of miR155 in the skin. This expression takes place mainly on Th1 and Th2 cells and is regulated by allergens and superantigens and is the highest especially during differentiation and activation of Th cells [37].

Thanks to miR-155 there is an increase in Th cell response by decreasing the expression of cytotoxic T lymphocyte-associated antigen 4 (CTLA-4) [37]. CTLA-4 is the key molecule that inhibits $T$ cell response, and its expression is inhibited by miR-155 in Th lymphocytes. CTLA4 molecule shows anti-proliferative function towards activated T cells, and increased expression of miR-155 enhances the proliferation of activated T cells CD4 [37]. CTLA-4 function as a potent negative regulator of $T$ cell responses has been confirmed in mice (CTLA- 4 knockout mice) that died prematurely due to multisystemic inflammation $[39,40]$. In animal models blocking of CTLA-4 provoked a much severe allergic reaction and inflammation [41], an increase in the number of eosinophils and IgE was observed, whereas an increase in the expression of CTLA-4 reduced severity of allergic pulmonary inflammation [42]. CTLA-4 can effectively inhibit inflammation in 
humans so it can be used in the treatment of chronic inflammatory diseases through abatacept-protein CTLA-4 immunoglobulin fusion [43]. It is also stated that 3'UTR transcript of CTLA-4 contains a constant evolutionary connecting section for miR-155. CTLA-4 protein expression in activated $T$ lymphocytes is inhibited by increased expression of miR-155. It is also suggested that miRNAs can control an early phase of inflammation by delaying the expression of CTLA-4 [37].

Evaluation of miRNAs in keratinocytes of AD patients showed elevated expression of miR-146a, miR-10b, miR10a, miR-10a*, miR-216, miR-921-1*, miR-454, miR-29b-1* and downregulation of miR-99a*, miR-34a*, miR34c-5p, miR-30a [44]. MiR-146a was one of the most upregulated molecules in the changed skin, and its increased expression was also found in the intact skin [44]. MiR-146a is another molecule that seems to play an important role in AD. It works by blocking the signal path NFKB (nuclear factor $\kappa$-light-chain-enhancer of activated $B$ cells) by direct action on IL-1 receptor-associated kinase 1 (IRAK1), TNF receptor-associated factor 6 (TRAF6) [45], v-rel avian reticuloendotheliosis viral oncogene homolog B (RELB) [46], and caspase recruitment domain (CARD10) [47].

In miR-146a-deficient mice the development of autoimmunity due to a failure in the activation of NFKB and signal transducer and activator of transcription 1 (STAT1) in T cells was observed [48, 49].

It has been detected that an increased level of miR146a controls chronic inflammation caused by increased levels of IFN- $\gamma$ and activation of NFKB in keratinocytes of AD patients. In this study, to identify molecules and cytokines that affect the expression of miR-146a keratinocytes were stimulated by various cytokines, heat-killed S. aureus or Toll-like receptor (TLR) ligands. Cytokines that caused the greatest expression of miR-146a were IL-1 $\beta$, TNF- $\alpha$, and IL-17A. A synergistic effect between these cytokines was also observed. It has been found that IFN- $\gamma$ did not induce the expression of miR-146a or inhibit the effect of TNF- $\alpha$ on the expression of miR-146a and had no effect on the action of IL-1 $\beta$ and IL-17A. Heat-killed S. aureus, IL-13, TLR2 and TLR4 ligands slightly upregulated, whereas IL-4 and IL-22 did not affect the expression of miR-146a [44].

MiR-146a results in suppression of such genes as CARD10, IRAK1, CCL5 and IL-8 stimulated and unstimulated keratinocytes. By comparing genes that undergo deregulations in $A D$ with a list of genes that are suppressed by miR-146a in keratinocytes that are stimulated by IFN- $\gamma$, seven overlapping genes have been found. From these genes CCL5, CCL8 and UBD (ubiquitin D) have been induced by IFN- $\gamma$ more than 10 times and suppressed by miR-146a more than 50\%. The analysis of these three genes has indicated that CCL5 mRNA possesses a potential binding site for miR-146a [44]. MiR-146a has caused a decrease in levels of CCL5, CXCL10 and IL-8 in keratinocytes stimulated by IFN- $\gamma$. Additionally, miR-146a has decreased the levels of IL- 6 and IL-8 in keratinocytes stimulated simultaneously by TNF- $\alpha$ and IFN- $\gamma$ [44].

Wanting to downregulate large numbers of genes in IFN- $\gamma$ stimulated keratinocytes, the researchers performed the pathway analysis which indicated the impact of miR-146a on the reduction in levels of phosphorylated and active NFKB subunit p65, and total p65, as well as on a slight reduction in NFkB p50 domain. Moreover, the expression of STAT1 protein was slightly decreased (approximately $20 \%$ ), and protein levels of the direct targets CARD10 and IRAK1 were suppressed by miR-146a [44].

It has been proved that the decrease in the expression of CARD10 results in downregulation of CCL5 mRNA (71.0\%) and its protein (58.1\%), and downregulation of UBD mRNA in IFN- $\gamma$ stimulated keratinocytes. It is suspected that the human CCL5 is well controlled by miR-146a as it is miR-146a' direct target. In addition, its expression is inhibited as miR-146a targets CARD10. Targeting of CARD10 and IRAK1 seems to be crucial because other mRNAs, such as UBD, CCL8 and IL-8, do not contain potential binding sites for miR-146a. The researchers suggest that CARD10 and UBD are novel factors that link IFN- $\gamma$ and NFKB pathways. Suppression of IRAK1 and CARD10 reduces the expression of IL-8 in keratinocytes stimulated by IFN- $\gamma$ [44]. Although miR-146a is expressed both in the epidermis and dermis, its direct targets IRAK1 and CARD10 are expressed higher in the epidermis, suggesting their important function in the regulation of inflammatory responses in keratinocytes [44].

Enhanced expression/inadequate suppression of $N F \kappa B$ in keratinocytes and uninvolved skin of $A D$ patients under the influence of miR-146a may be caused by epigenetic changes in miR-146a promoter or could represent a different activation state of AD patients' keratinocytes. Increased expression of miR-146a has also been found in psoriasis, indicating this molecule' participation in the inflammatory response in the skin [8]. The feedback of a loop effect has also been found: higher production of NFKB causes higher expression of miR-146a, which targets several modulators of NFKB pathway including IRAK1, TRAF6, RELB and CARD10 in different cell types [45-47].

In another study the expression of miRNAs has been determined in urine and serum in AD pediatric population. By means of the Taqman-based array and specified miRNAs expression profiles, 166 of miRNAs have been detected in urine and 255 in serum, in which 9 miRNAs have been upregulated (miR-205, miR-539, miR-122, miR203, miR-483-5p, miR-134, let-7g, miR-495, miR-642) and 1 downregulated (miR-590-5p) in serum and 10 have been upregulated (MammU6, miR-142-3p, miR-20a, miR-548c-3p, miR-205, miR-19a, miR-483-5p, miR-222, miR-92a, miR-548a-3p) and 7 downregulated (miR-203, miR-125a-5p, miR-886-3p, miR-184, miR-886-5p, miR-26a, miR-194) in urine compared to the healthy population. Among tested miRNAs only miR-203, miR-483-5p and 
miR205 have been present in urine and serum. In addition, miR-483-5p and miR-205 have been upregulated in serum and urine, and miR-203 has been upregulated in serum and downregulated in urine of the children with $\mathrm{AD}[50]$.

More accurate analysis using quantitative real-time PCR has been performed on upregulated expression levels of miRNAs including miR-203, miR-205, miR-4835p, miR-134 and miR-122. These miRNAs have been described as highly expressed in AD lesional skin and involved in immuno-regulatory network [8, 51-54]. In this analysis, miR-483-5p and miR-203 have been significantly upregulated in serum of AD children compared to the controls but it has not indicated a significant difference in the expression of miR-122, miR-134 and miR-205 in serum compared to the controls. Patients with a higher level of IgE in serum had a significantly higher expression of miR-203 in serum compared to the controls, whereas AD patients with a normal level of IgE had no significant difference compared to the controls. Yet, patients with higher or normal IgE levels had a significantly higher miR-483-5p expression in serum compared to the controls [50].

While comparing other characteristics, such as ages, gender, SCORAD and the number of eosinophils, no significant differences in the expression of miR-483-5p and miR-203 in serum of patients with AD have been found. However, a relationship between upregulation of miR-483$5 p$ in serum and other atopic diseases in patients with $A D$, like rhinitis and/or asthma, has been detected [50].

It has also been stated that only miR-203 was significantly downregulated in urine of the children with AD compared to the controls, whereas miR-205 and miR-483-5p in urine did not show any significant changes between these two groups. Patients with a higher level of IgE in serum had a significantly lower expression of miR-203 in urine compared to the controls, however, AD patients with a normal level of IgE had no significant difference compared to the controls. No differences have been found in the comparison concerning the expression of miR-203 in urine of the children with AD when taking into account such features as: age, gender, SCORAD, number of eosinophils and other concurrent atopic conditions [50].

The analysis has confirmed only two miRNAs (miR203 and miR-483-5p) which were statistically upregulated in serum of the children with AD. MiR-203 was originally described as a molecule specific for keratinocytes and being increasingly expressed in skin lesions of psoriatic patients, but not in $A D$ patients (adult population) [8]. There was a higher expression of miR-203 in serum in the children with eAD (extrinsic AD). It has also been shown that miR-483-5p molecule is upregulated in serum of patients with iAD (intrinsic $A D$ ) and eAD, which may lead to indicate this miRNAs as a marker for AD independent of the level of total lgE in serum. Increased expres- sion of miR-483-5p in serum of children with other atopic diseases has been detected as well. This may suggest that miR-483-5p are the molecules involved in multiorgan atopic reactions [8]. Atopic dermatitis patients with an increased expression of miR-203 in serum showed a decreased expression of miR-203 in urine, which also was associated with a higher level of total IgE and increased inflammation of the skin. Lower levels of miR-203 in urine have been reported in patients with eAD compared to the ones with $\mathrm{iAD}$. This finding suggests that upregulated miR-483-5p in serum may be an indicator of other atopic conditions in the children with AD while downregulated miR-203 in urine may serve as a biomarker for the severity of inflammation in AD children. The expression of miRNAs in serum and urine of $A D$ patients in the study on children population differs from the one which describes skin lesions in the adults [50].

\section{Conclusions}

Literature reports have already indicated that miRNAs can be used in the treatment of various diseases [55]. Development of drugs may rely on the modulation of the expression of miRNAs by means of oligonucleotides that combine with miRNA molecules, inhibiting the use of primiRNAs and pre-miRNAs, as well as affecting miRNAs in a protein complex.

In the case of HCV infection, the studies on humans have entered the second clinical phase and indicated that subcutaneous administration of miravirsen, oligonucleotide inhibiting the activity of miRNAs-122, necessary for proliferation of HCV-RNA, results in a long-lasting effect of reducing HCV-RNA levels in patients [56].

Chemokine CCL22, which concentration is increased in $A D$, results in migration and increase in the number of Th2 cells in the skin. CCL22 predominantly affects CD4 T cells that have a skin-homing receptor, known as cutaneous lymphocyte-associated antigen (CLA) [57]. CCL22 levels are correlated with the severity of disease in patients with $A D$ [58]. In the study on mouse models of AD Yoon et al. [59] designed miRNAs that interfered with CCL22 mRNAs. Salmonella typhimurium was used as the vector and it was administered orally. Clinical improvement in mice in the range of skin lesions, reduction in scratching reflex, decreased levels of CCL22 and IL-4 as well as increase in the level of IFN- $\gamma$ were described [59]. IL-4 is the key factor which causes switching B cells subtype by producing IgE [60], whereas IFN- $\gamma$ inhibits the secretion of IgE [61].

It should be noticed that the studies on miRNAs in AD were carried out on relatively small and heterogeneous groups of patients so their results are not conclusive. They may depend on the patient's age, severity of lesions, levels of total lgE as well as differential expression of miRNAs in the analyzed samples: skin, serum or urine. Therefore, further research should be homogeneous and 
made on large groups of patients with comparable and standardized test methods. Despite these inconsistencies, miRNA molecules are very promising and may provide another source of diagnosis and treatment in AD.

\section{Conflict of interest}

The authors declare no conflict of interest.

\section{References}

1. Carthew RW, Sontheimer EJ. Origins and mechanisms of miRNAs and siRNAs. Cell 2009; 136: 642-55.

2. Reinhart BJ, Weinstein EG, Rhoades MW, et al. MicroRNAs in plants. Genes Dev 2002; 16: 1616-26.

3. Krol J, Loedige I, Filipowicz W. The widespread regulation of microRNA biogenesis, function and decay. Nat Rev Genet 2010; 11: 597-610.

4. Winter J, Jung S, Keller S, et al. Many roads to maturity: microRNA biogenesis pathways and their regulation. Nat Cell Biol 2009; 11: 228-34.

5. Rebane A, Akdis CA. MicroRNAs: essential players in the regulation of inflammation. J Allergy Clin Immunol 2013; 132: 15-26.

6. Makeyev EV, Maniatis T. Multilevel regulation of gene expression by microRNAs. Science 2008; 319: 1789-90.

7. Djuranovic S, Nahvi A, Green R. A parsimonious model for gene regulation by miRNAs. Science 2011; 331: 550-3.

8. Sonkoly E, Wei T, Janson PC, et al. MicroRNAs: novel regulators involved in the pathogenesis of psoriasis? PLoS One 2007; 2: e610.

9. Bartel DP. MicroRNAs: target recognition and regulatory functions. Cell 2009; 136: 215-33.

10. Chiang HR, Schoenfeld LW, Ruby JG, et al. Mammalian microRNAs: experimental evaluation of novel and previously annotated genes. Genes Dev 2010; 24: 992-1009.

11. Meunier J, Lemoine F, Soumillon M, et al. Birth and expression evolution of mammalian microRNA genes. Genome Res 2013; 23: 34-45.

12. Lee RC, Feinbaum RL, Ambros V. The C. elegans heterochronic gene lin-4 encodes small RNAs with antisense complementarity to lin-14. Cell 1993; 75: 843-54.

13. Jinnin M. Various applications of microRNAs in skin diseases. J Dermatol Sci 2014; 74: 3-8.

14. Bushati N, Cohen SM. microRNA functions. Annu Rev Cell Dev Biol 2007; 23: 175-205.

15. Leung AK, Sharp PA. MicroRNA functions in stress responses. Mol Cell 2010; 40: 205-15.

16. O'Connell RM, Rao DS, Baltimore D. microRNA regulation of inflammatory responses. Annu Rev Immunol 2012; 30: 295312.

17. Chen X, Ba Y, Ma L, et al. Characterization of microRNAs in serum: a novel class of biomarkers for diagnosis of cancer and other diseases. Cell Res 2008; 18: 997-1006.

18. Zen K, Zhang CY. Circulating microRNAs: a novel class of biomarkers to diagnose and monitor human cancers. Med Res Rev 2012; 32: 326-48.

19. Garbacki N, Di Valentin E, Huynh-Thu VA, et al. MicroRNAs profiling in murine models of acute and chronic asthma: a relationship with mRNAs targets. PLoS One 2011; 6: e16509.
20. Fallon PG, Mangan NE. Suppression of Th2-type allergic reactions by helminth infection. Nat Rev Immunol 2007; 7: 220-30.

21. Zheng Y, Cai X, Bradley JE. microRNAs in parasites and parasite infection. RNA Biol 2013; 10: 371-9.

22. Cortez MA, Bueso-Ramos C, Ferdin J, et al. MicroRNAs in body fluids: the mix of hormones and biomarkers. Nat Rev Clin Oncol 2011; 8: 467-77.

23. Cai Y, Fleming C, Yan J. New insights of T cells in the pathogenesis of psoriasis. Cell Mol Immunol 2012; 9: 302-9.

24. Sonkoly E, Stahle M, Pivarcsi A. MicroRNAs: novel regulators in skin inflammation. Clin Exp Dermatol 2008; 33: 312-5.

25. Zibert JR, Løvendorf MB, Litman T, et al. MicroRNAs and potential target interactions in psoriasis. J Dermatol Sci 2010; 58: 177-85.

26. Ichihara A, Jinnin M, Yamane K, et al. microRNA-mediated keratinocyte hyperproliferation in psoriasis vulgaris. $\mathrm{Br}$ J Dermatol 2011; 165: 1003-10.

27. Guo S, Zhang W, Wei C, et al. Serum and skin levels of miR369-3p in patients with psoriasis and their correlation with disease severity. Eur J Dermatol 2013; 23: 608-13.

28. LeRoy EC, Medsger Jr TA. Criteria for the classification of early systemic sclerosis. J Rheumatol 2001; 28: 1573-6.

29. Zhu H, Li Y, Qu S, et al. MicroRNA expression abnormalities in limited cutaneous scleroderma and diffuse cutaneous scleroderma. J Clin Immunol 2012; 32: 514-22.

30. Sing T, Jinnin M, Yamane K, et al. microRNA-92a expression in the sera and dermal fibroblasts increases in patients with scleroderma. Rheumatology (Oxford) 2012; 51: 1550-6.

31. Kajihara I, Jinnin M, Yamane K, et al. Increased accumulation of extracellular thrombospondin-2 due to low degradation activity stimulates type I collagen expression in scleroderma fibroblasts. Am J Pathol 2012; 180: 703-14.

32. Maurer B, Stanczyk J, Jüngel A, et al. MicroRNA-29, a key regulator of collagen expression in systemic sclerosis. Arthritis Rheum 2010; 62: 1733-43.

33. Honda N, Jinnin M, Kajihara I, et al. TGF-beta-mediated downregulation of microRNA-196a contributes to the constitutive upregulated type I collagen expression in scleroderma dermal fibroblasts. J Immunol 2012; 188: 3323-31.

34. Bieber T. Atopic dermatitis. N Engl J Med 2008; 358: 1483-94.

35. Cookson WO, Moffatt MF. The genetics of atopic dermatitis. Curr Opin Allergy Clin Immunol 2002; 2: 383-7.

36. Vennegaard MT, Bonefeld CM, Hagedorn PH, et al. Allergic contact dermatitis induces upregulation of identical microRNAs in humans and mice. Contact Dermatitis 2012; 67: 298-305.

37. Sonkoly E, Janson P, Majuri ML, et al. MiR-155 is overexpressed in patients with atopic dermatitis and modulates T-cell proliferative responses by targeting cytotoxic T lymphocyte-associated antigen 4. J Allergy Clin Immunol 2010; 126: 581-9.e1-20.

38. Sonkoly E, Pivarcsi A. Advances in microRNAs: implications for immunity and inflammatory diseases. J Cell Mol Med 2009; 13: 24-38.

39. Waterhouse P, Penninger JM, Timms E, et al. Lymphoproliferative disorders with early lethality in mice deficient in CTLA-4. Science 1995; 270: 985-8.

40. Tivol EA, Borriello F, Schweitzer AN, et al. Loss of CTLA-4 leads to massive lymphoproliferation and fatal multiorgan tissue destruction, revealing a critical negative regulatory role of CTLA-4. Immunity 1995; 3: 541-7.

41. Hellings PW, Vandenberghe P, Kasran A, et al. Blockade of CTLA-4 enhances allergic sensitization and eosinophilic 
airway inflammation in genetically predisposed mice. Eur J Immunol 2002; 32: 585-94.

42. Jen KY, Campo M, He H, et al. CD45RB ligation inhibits allergic pulmonary inflammation by inducing CTLA4 transcription. J Immunol 2007; 179: 4212-8.

43. Abrams JR, Lebwohl MG, Guzzo CA, et al. CTLA4lg-mediated blockade of T-cell costimulation in patients with psoriasis vulgaris. J Clin Invest 1999; 103: 1243-52.

44. Rebane A, Runnel T, Aab A, et al. MicroRNA-146a alleviates chronic skin inflammation in atopic dermatitis through suppression of innate immune responses in keratinocytes. J Allergy Clin Immunol 2014; 134: 836-47.e11.

45. Taganov KD, Boldin MP, Chang KJ, Baltimore D. NF-kappaBdependent induction of microRNA miR-146, an inhibitor targeted to signaling proteins of innate immune responses. Proc Natl Acad Sci U S A 2006; 103: 12481-6.

46. Etzrodt M, Cortez-Retamozo V, Newton A, et al. Regulation of monocyte functional heterogeneity by miR-146a and Relb. Cell Rep 2012; 1: 317-24.

47. Crone SG, Jacobsen A, Federspiel B, et al. microRNA-146a inhibits $G$ protein-coupled receptor-mediated activation of NF-kappaB by targeting CARD10 and COPS8 in gastric cancer. Mol Cancer 2012; 11: 71.

48. Lu LF, Boldin MP, Chaudhry A, et al. Function of miR-146a in controlling Treg cell-mediated regulation of Th1 responses. Cell 2010; 142: 914-29.

49. Boldin MP, Taganov KD, Rao DS, et al. miR-146a is a significant brake on autoimmunity, myeloproliferation, and cancer in mice. J Exp Med 2011; 208: 1189-201.

50. Lv Y, Qi R, Xu J, et al. Profiling of serum and urinary microRNAs in children with atopic dermatitis. PLoS One 2014; 9: e115448.

51. Wang G, Tam LS, Li EK, et al. Serum and urinary free microRNA level in patients with systemic lupus erythematosus. Lupus 2011; 20: 493-500.

52. Wu TH, Pan CY, Lin MC, et al. In vivo screening of zebrafish microRNA responses to bacterial infection and their possible roles in regulating immune response genes after lipopolysaccharide stimulation. Fish Physiol Biochem 2012; 38: 1299 310.

53. Li A, Song W, Qian J, et al. MiR-122 modulates type I interferon expression through blocking suppressor of cytokine signaling 1. Int J Biochem Cell Biol 2013; 45: 858-65.

54. Zhang X, Wang H, Zhang S, et al. MiR-134 functions as a regulator of cell proliferation, apoptosis, and migration involving lung septation. In Vitro Cell Dev Biol Anim 2012; 48: 131-6.

55. Cardona ID, Cho SH, Leung DY. Role of bacterial superantigens in atopic dermatitis: implications for future therapeutic strategies. Am J Clin Dermatol 2006; 7: 273-9.

56. de Jong YP, Jacobson IM. Antisense therapy for hepatitis $C$ virus infection. J Hepatol 2014; 60: 227-8.

57. Campbell DE, Kemp AS. Cutaneous lymphocyte-associated antigen expression in children with atopic dermatitis and non-atopic healthy children. Pediatr Allergy Immunol 1999; 10: 253-7.

58. Nakazato J, Kishida M, Kuroiwa R, et al. Serum levels of Th2 chemokines, CCL17, CCL22, and CCL27, were the important markers of severity in infantile atopic dermatitis. Pediatr Allergy Immunol 2008; 19: 605-13.

59. Yoon WS, Lee SS, Chae YS, Park YK. Therapeutic effects of recombinant Salmonella typhimurium harboring CCL22 miRNA on atopic dermatitis-like skin in mice. Exp Mol Med 2011; 43: 63-70.
60. Fiset PO, Leung DY, Hamid Q. Immunopathology of atopic dermatitis. J Allergy Clin Immunol 2006; 118: 287-90.

61. Herberth G, Heinrich J, Röder S, et al. Reduced IFN-gammaand enhanced IL-4-producing CD4+ cord blood T cells are associated with a higher risk for atopic dermatitis during the first 2 yr of life. Pediatr Allergy Immunol 2010; 21: 5-13. 